\title{
A Novel Model for SOAs in WDM Networks
}

\author{
W. Mathlouthi (1), P. Lemieux (1), M. Salsi (2), A. Vannucci (2), A. Bononi (2) and L. A. Rusch (1) \\ 1: COPL, ECE Dept., Université Laval, Sainte-Foy, Québec G1K 7P4, Canada. Email : rusch@gel.ulaval.ca \\ 2: Dipartimento Di Ingenieria dell’Informazione, Università di Parma, I-43100, Italy. Email: salsi@tlc.unipr.it
}

\begin{abstract}
We present a novel state-variable model for semiconductor optical amplifiers, amenable to block diagram implementation, and with fast execution times.
\end{abstract}

\section{INTRODUCTION}

Semiconductor optical amplifiers (SOA) are becoming a key technology for future optical networks. SOA models can be divided in two categories: space-resolved, numerically intensive models and analytical, simplified models. We present a novel simplified analytical model, originally inspired by the work of Agrawal et al. [1], which dramatically improves execution times when extensive Monte-Carlo simulation for statistical signal analysis and bit-error rate (BER) estimation are performed. In addition, for the first time, the model accounts for the influence of amplified spontaneous emission (ASE) noise and scattering loss on the gain dynamics. Our model is based on a single state variable, the reservoir, which allows a fast calculation of the response of the SOA to a wavelength division multiplexed (WDM) optical signal. In this work, we neglect ultrafast intraband phenomena such as carrier heating and spectral hole burning, which is a suitable approximation for bit rates up to $10 \mathrm{Gbit} / \mathrm{s}$ per channel. We also assume the WDM channels are spaced apart far enough to neglect carrier density pulsations that cause four-wave mixing. Our results are compared to experiments and to simulation results obtained from the accurate space resolved Connelly model [2].

\section{SOA RESERVOIR MODEL}

We assume here a travelling-wave SOA, with a cavity volume $V=A L$, where $A$ is the active waveguide area and $L$ is the total SOA length. Define the SOA reservoir as:

$$
r(t)=A \int_{0}^{L} N(z, t) d z
$$

where $N(z, t)$ is the carrier density at coordinate $z$. If we assume a linear dependence on $N$ of the material gain coefficient $g_{m}$ and the scattering losses $\alpha$, as done, e.g., in [2] (eqs. (14) and (18)), we obtain a straightforward expression of the inputoutput net gain (including scattering losses) for the k-th channel in terms of the reservoir: $G_{k}(r)=\exp \left\{a_{k}\left(r(t)-r_{0 k}\right)\right\}$. Here, the parameters $a_{k}$ and $r_{0 k}$ are functions of the wavelength $\lambda_{k}$, and can be derived from a linearization of the net gain. Under such hypotheses, we can integrate the SOA rate equation ([2], eq. (48)) so as to obtain the Reservoir equation:

$$
\begin{aligned}
\frac{d r}{d t}= & \frac{I}{q}-\frac{r}{\tau}-\sum_{k=1 \text { to } N_{S i g}} Q_{k}^{I N}\left(G_{k}(r)-1\right) \\
& -4 \Delta v_{A S E} \sum_{j=1 \text { to } N_{A S E}} n_{s p, j}(r)\left(G_{j}(r)-1-\ln G_{j}(r)\right)
\end{aligned}
$$

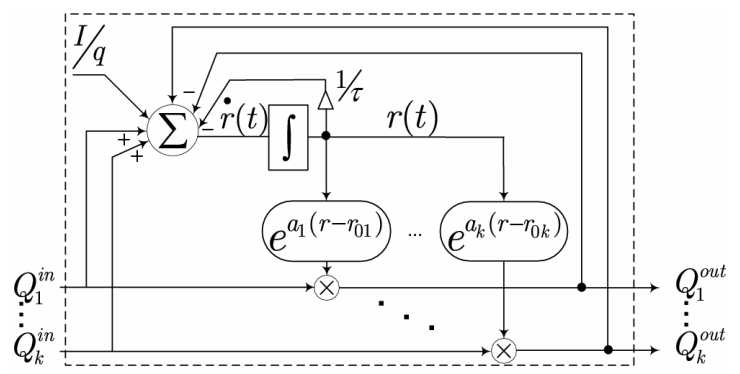

Fig. 1. Block diagram of the reservoir model

where $I$ is the injection current, $q$ is the electron charge, $\tau$ is the fluorescence time, $Q_{k}^{I N}$ are the input fluxes (in photons/second) and the ASE is included using the length averaged spontaneous emission factor $n_{s p}$ and ASE channel spacing $\Delta v_{A S E}$. The study of the SOA dynamics can be reduced to the solution of the single ordinary differential equation (2). The state variable $r(t)$ is called here the reservoir, since, being proportional to the integrated carrier density, it plays the same role as the reservoir of excited erbium ions in an erbiumdoped fibre amplifier (EDFA) [3]. Once the state variable dynamics are found, the dynamics of all output WDM channels are also obtained as:

$$
Q_{k}^{O U T}(t)=Q_{k}^{I N}(t) G_{k}(r(t))
$$

Quite interestingly, then, the SOA for WDM operation admits almost the same block diagram description as that of an EDFA suggested by eq. (5) in [3]. Such a novel SOA block diagram is shown in Fig. 1 (without ASE for ease of drawing). It is easy to understand that such single variable (and single equation) model becomes more and more efficient with respect to a space-resolved model when the number of WDM channels increases.

Note that eq. (2) accounts for both ASE and scattering losses, since the net gain $G_{k}(r)$ appears. It can be shown that the approximation entailed in (2) brings about a slight overestimation of $r(t)$, a fact that we heuristically account for by slightly decreasing the value of the fluorescence time when fitting experimental results [4].

\section{EXPERIMENTAL RESULTS AND SIMULATIONS}

Our experimental setup consists of a tuneable laser source followed by an external Mach-Zehnder modulator at $1 \mathrm{~Gb} / \mathrm{s}$. The modulated signal enters an SOA (Optospeed model 1550MRI X1500) with an input current of $500 \mathrm{~mA}$. The signal is then photodetected without any optical filtering. The same system is simulated using both a dynamic version of the 


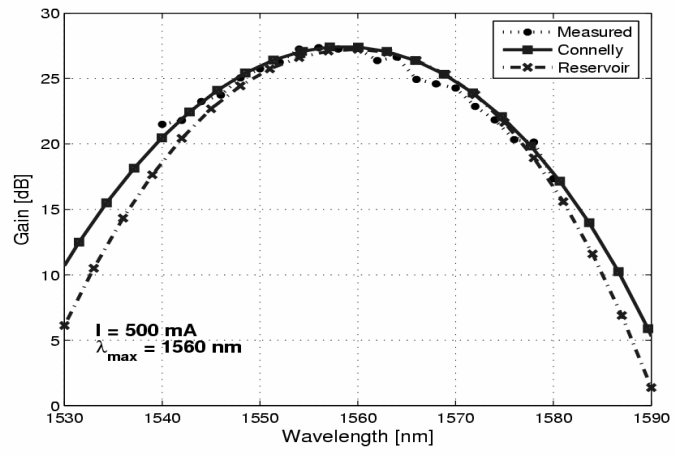

(a)

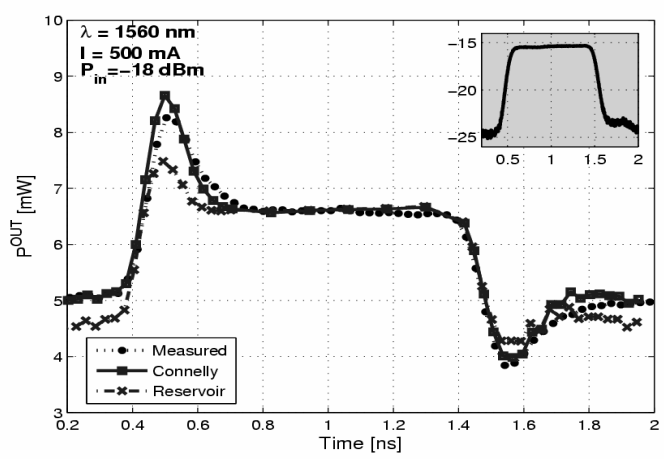

(c)

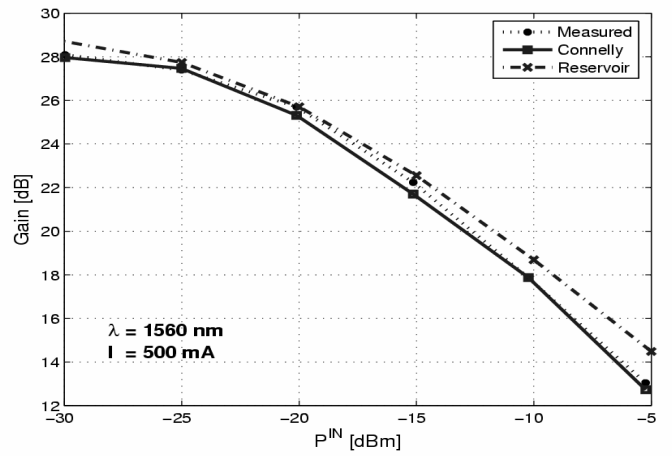

(b)

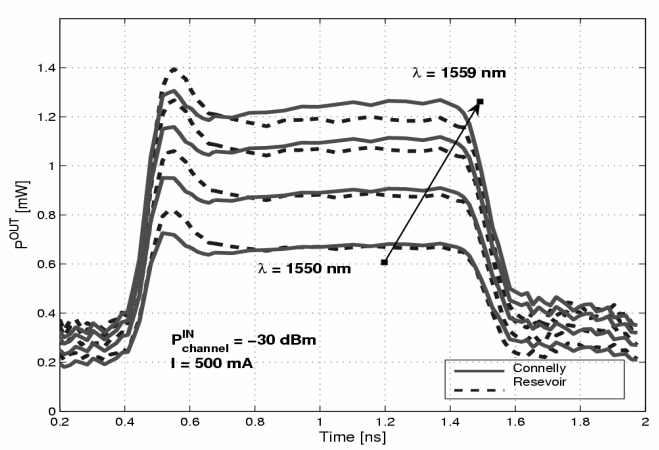

(d)

Fig. 2. Amplifier gain versus wavelength (a) and versus input power (b). Response of the amplifier to a square wave input with single channel input (c) and with 4 channels input (d). From (a) to (c) there are experimental results, space-resolved simulation (Connelly) and our model (Reservoir); in (d) there are the two simulations only.

space-resolved Connelly model [2] and using our Reservoir model. Figure 2(a-c) shows a comparison of experiments and simulations (Connelly and Reservoir models). The three plots represent, respectively, the SOA total gain curve versus wavelength (using an average input power of $-18 \mathrm{dBm}$ ), the gain saturation curve versus input power (at $1560 \mathrm{~nm}$ ), and the dynamic response of the SOA to a square wave input (visible in the inset). It is possible to see that both simulation approaches agree quite well with experimental results, but the Reservoir model runs 20 times faster than the space-resolved model. To verify the efficiency of our model in a wider range of simulation scenarios we investigated the case of WDM signal amplification. In figure 2(d) we report the response of the SOA to a four signal WDM input (wavelength spacing of $3 \mathrm{~nm}$, from $1550 \mathrm{~nm}$ to $1559 \mathrm{~nm}$ ) with synchronous OOK modulation. In this figure the two simulation approaches only are compared. While the simulation times of the space-resolved Connelly model considerably increase, those of the Reservoir model are almost insensitive to the number of WDM channels.

\section{CONCLUSION}

A novel SOA dynamic model, based on the reservoir state variable, has shown good accuracy and computation times 20 times shorter than the space-resolved models, for single channel transmission. Since computation times are little affected by the number of channels, the reservoir model is especially efficient for modeling SOAs in WDM optical networks simulations requiring intense computation as, e.g., in BER estimation. In addition, such novel model allows analytic insight into the SOA operation and flexibility in the implementation. In fact, a number of variants can be introduced on the reservoir model, which we explore in [4], like using a single equivalent ASE channel, to further speed up calculations, or modeling a SOA with multiple reservoirs, to increase precision at the expense of calculation times.

\section{REFERENCES}

[1] G. P. Agrawal et al., "Self-Phase Modulation and Spectral Broadening of Optical Pulses in Semiconductor Laser Amplifier," IEEE J. Quantum Electronics, vol. 25, pp. 2297-2306, 1989.

[2] M. J. Connelly, "Wideband Semiconductor Optical Amplifier SteadyState Numerical Model," IEEE J. Quantum Electronics, vol. 37, pp. 439-447, 2001.

[3] A. Bononi et al., "Doped-Fiber Amplifier Dynamics: A system Perspective," IEEE J. Lightwave Technology, vol. 16, pp. 945-956, 1998.

[4] W. Mathlouthi et al., "Fast and Efficient Dynamic WDM Semiconductor Optical Amplifier Model," IEEE J. Lightwave Technology, submitted. 